\title{
Another Notch in neurogenesis
}

During the development of the central nervous system (CNS) or peripheral nervous system (PNS) in Drosophila melanogaster, the neural progenitors (neuroblasts, NB) or the sensory organ precursors, respectively, give rise to lineages of neural and glial cells. Many of the factors that control asymmetric division of the these cells and the development of the neural lineages have been determined. However, previous work has not focused on the mechanisms behind glial cell development. Recent work by Udolph et al. (Development, 128, 1457-1466; 2001) and Van De Bor et al. (Development 128, 1381-1390; 2001) studied the role of Notch - a protein known to be involved in cell communication and with a presumed role in gliogenesis - in this process.

CNS glial cells develop from glioblast or neuroglioblast (NGB) lineages derived from the neuroectoderm or the mesoectoderm, whereas PNS glial cells are derived from mixed lineages. During early lineage development, some NGB lineages bifurcate to produce a glioblast and a neuroblast in an asymmetric manner. Neuroectoderm-derived glioblasts express glial cells missing $(\mathrm{gcm})$, which acts to switch cells between neuronal and glial cell fates. As there are many different glial cell progenitors, Udolph et al. studied the NB1-1A abdominal lineage and showed that it does not bifurcate into more specialized secondary progenitors. Instead, the glial cells share a sibling cell fate relationship with neurons. Without Notch, the glial cells are lost and the neuronal cells are duplicated (see picture). In addition, Notch positively regulates the expression of $\mathrm{gcm}$.

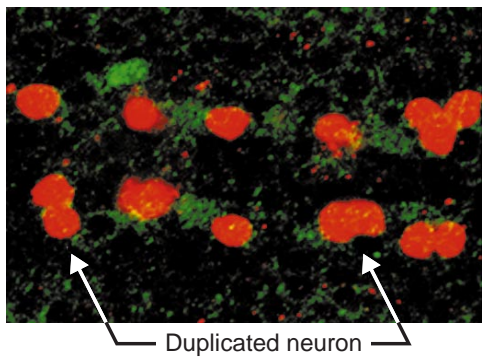

THE COMPANY OF BIOLOGISTS

In contrast, Van De Bor et al. studied gliogenesis in the PNS, where gain-of-function Notch mutations also act as a genetic switch between neuronal and glial cell fates. Interestingly, they show that Notch represses neurogenesis or gliogenesis within certain cell types and, in contradiction of the work of Udolph et al., that it represses the expression of $\mathrm{gcm}$.

These studies indicate that Notch regulates by controlling, either positively or negatively, the glial master regulator $\mathrm{gcm}$. Although the precise role of Notch, and its relationship with $\mathrm{gcm}$ expression, seems to depend on the neuronal lineage studied, both pieces of work uncover some of the mechanisms used to control the fate of glial cells.

SARAH GREAVES

virus, indicating either that the virus triggered caveolar internalization or that it somehow facilitated the identification of such an event. Viruses then moved to a pre-existing, caveolin-positive, internal structure that was relatively immobile. This compartment did not contain markers for the Golgi complex, early endosomes, lysosomes or the ER. In addition, this compartment was not in contact with the extracellular medium and was of neutral internal $\mathrm{pH}$. The authors named this structure a caveosome.

Although delivery to caveosomes was slow and virions remained there for several hours, the compartment underwent a remarkable transformation - after several hours it became motile and underwent fusion and fission reactions. Especially striking was the departure of viruses from caveosomes and their segregation from caveolin, which remained associated with caveosomes. Viruses used microtubules to traverse to perinuclear sites, and this step was necessary both for productive infection and for ER delivery. Within the ER, viruses occupied a caveolin-lacking ER compartment that seemed to represent smooth ER.

Conversion of a caveosome into a motile compartment requires recruitment or activation of organelle-associated molecular motors. However, sorting of SV40 requires more - proteins are needed to segregate departing cargoes (SV40) from resident proteins (caveolins). Moreover, the departing vesicles and tubules must have targetspecifying molecules on their surfaces. As caveosomes themselves seem not to fuse with the ER, perhaps they are the infrequent target of vesicles that carry with them the transport machinery to mediate delivery to the ER. Such a vesicle would have the characteristics of the intermediate compartment between ER and Golgi that is equipped to recycle certain exported proteins back to the ER.

Is caveolar uptake a constitutive process in the absence of SV40 infection? Although this remains to be determined, Nichols and colleagues have found that internalization of certain GPI-linked proteins, which is presumably mediated by caveolae (although this remains to be proved), also bypasses conventional endocytic compartments. Whereas the GPI-linked folate receptor is internalized through recycling endosomes ${ }^{10}$, Nichols and colleagues have shown that GPI-linked CD59 and GPI-GFP are continuously transported between the cell surface and the Golgi complex. Like caveolar internalization of SV40, internalization of GPI-GFP was slow - a given GPI-linked protein had a residence time of $200 \mathrm{~min}$ at the cell surface. Once transported to the Golgi complex, GPI-GFP then returned relatively rapidly to the cell surface. Export from the Golgi complex required intact microtubules, and import was blocked at $20^{\circ} \mathrm{C}$. GPI-GFP was internalized into the same compartments as a large fraction of the glycosphingolipid-binding, cholera and Shiga toxin B subunits, and this process was shown to be independent of both the clathrin-endocytic machinery and Rab5 GTPase function ${ }^{6}$. As expected for a caveolae-mediated process, uptake was inhibited by cholesterol depletion.

These recent reports raise many immediate questions. Does GPI-GFP use caveolae for internalization and does it enter caveosomes? Determining whether it colocalizes with caveolin and SV40 should resolve these matters. If GPI-GFP does enter caveosomes, it is not expected to have a long residence time in that compartment, as it does not accumulate there. The transport carriers that internalize GPI-GFP contained fluid-phase markers, unlike those that internalize SV40 (ref. 7). Such markers could have been excluded by a virus that is enveloped by the caveolar membrane during internalization. As fluid-phase markers do not usually enter the Golgi complex or the ER, their presence in vesicles carrying GPI-GFP indicates that GPI-GFP may be sorted within some intermediate compartment before it is delivered to the Golgi complex. If a common caveosome is used by SV40 and GPI-GFP, that compartment 